\title{
A Method to Improve the Performance of Gold Nanoparticle Suspensions for Trace Detection of 16 EPA Priority PAHs
}

\author{
Anna Kolomijeca ${ }^{1,2}$ \\ ${ }^{1}$ Department of Ecology and Evolutionary biology, University of Toronto, Canada \\ ${ }^{2}$ Department of Mechanical and Industrial Engineering, University of Toronto, Canada
}

Copyright $\bigcirc 2019$ by authors, all rights reserved. Authors agree that this article remains permanently open access under the terms of the Creative Commons Attribution License 4.0 International License

\begin{abstract}
The growing demand for fast, simple and inexpensive analytical techniques to investigate environmental pollution has resulted in a large number of studies where surface enhanced Raman spectroscopy is used to detect environmental contaminants. However, extraction of weak Raman spectra from complex background signals remains challenging. In this current work, the author has presented a method to improve the performance of gold nanoparticles suspensions for trace detection of the 16 EPA priority PAHs. The gold nanoparticles in suspension are first mixed with the analyte in the liquid phase and then transferred to a solid state by drying (as opposed to liquid sample + dry substrate or liquid sample + liquid substrate). This method allows for a complete background signal removal (including fluorescence and signal from the substrate), strong Raman signal enhancement from the analyte and a reduction in substrate preparation time. Performance of a developed substrate $(\mathrm{CNP}-\mathrm{Au})$ was compared with a commercially available 3D substrate (Ram-SERS-Au) by measuring of all 16 EPA priority PAHs. The developed CNP-Au substrate exhibited experimentally obtained LODs three orders of magnitude lower than Ram-SERS-Au, down to the range of $1 \mathrm{ppb}$. The standard deviation of signal reproducibility for CNP-Au is $14 \%$. Ram-SERS-Au and $\mathrm{CNP}-\mathrm{Au}$ were characterized using SEM imaging.
\end{abstract}

Keywords SERS Suspensions, Priority PAHs, Gold Nanoparticles, Raman

\section{Introduction}

Polycyclic aromatic hydrocarbons (PAHs) are common environmental pollutants with toxic, mutagenic and/or carcinogenic properties [1]. Identification of PAHs in environmental samples is essential for understanding their prevalence and impacts. Raman spectroscopy allows one to obtain a "fingerprint" of information of a chemical(s) within seconds. However, when measuring PAHs at environmentally-relevant concentrations, the signal is too weak to be detected [2] due to a low Raman scattering cross section. The application of Surface Enhanced Raman Spectroscopy (SERS) can increase measurement sensitivity up to a factor of $10^{7}$ [3]. The electromagnetic enhancement mechanism is dominant in SERS [4] and occurs due to the concentration of electromagnetic field associated with light in nanostructured electronically-conducting materials (such as silver or gold), followed by excitation of surface plasmons (SP) that resonantly emit radiation from nearby molecules [5]. Nanoparticles morphology and degree of aggregation will dictate SERS efficiency, since junctions between the particles will serve as SERS hot spots, enabling single molecule detection [6]. Typically three types of nanoparticles are used for SERS: nanospheres, nanotriangles and nanostars [7], as well as three types of substrates: (1) metal nanoparticles in suspension [8]; (2) metal nanoparticles immobilized on solid-support substrate (prepared by bottom-up methods, such as wet chemistry [6], [9], [10]) and (3) nanostructures fabricated directly on solid-support substrates (generally prepared by a top-down methods, such as lithography-based fabrication [11], [12], [13] or templating [14]). Most of the studies that have been done on PAH detection with SERS apply solid-support substrates prepared by bottom-up approach [15], [16], [9], [17], [18], [19], [20], [21], [22], [23] due to the low production costs, good substrate performance and high reproducibility. However, this requires preparation of a new substrate before each measurement, which can be time-consuming. Shi et al [24] applies gold nanoparticles suspension to detect five different PAHs. However, presented measurements strongly suffer from background 
noise. In fact, extracting weak Raman spectra from a complex background signal has been a significant challenge for all above studies. For example, in the studies of Bao et al [21] and Shi et al [24], [25], the signal from the analyte (in LOD concentrations) cannot be seen without the magnification of the specific spectral area due to strong background noise. This causes problems measuring untargeted samples, as it is important to observe more than one peak to determine chemicals present.

Several methods exist to extract the Raman spectra from the background noise: a) reducing fluorescence of the sample by photobleaching [26], fluorescence quenching[27], sample washing or filtration [28] (only possible for some samples), b) manipulating Raman scattering properties, such as using longer wavelengths, or anti-Stokes Raman spectroscopy (which produces a lower Raman signal response); c) application of computational methods, such as baseline correction, background extraction (which can be efficient for the initial substrate-spectra removal, but less efficient for autofluorescence correction); d) application of dual laser measurements in combination with computational technique, such as shifted excitation Raman difference spectroscopy (SERDS) [29] (which requires sophisticated equipment).

Because the current study deals with persistent organic pollutants that are relatively stable, the author applies a heat treatment technique (excess liquid evaporation) on a sample to completely eliminate fluorescence and background noise.

Typically, an analyte is added to a nanoparticle suspension or applied on the top of solid-support substrate and measured as liquid + liquid or solid + liquid (in case liquid sample is dried on the top of the solid substrate, the sample will cover only a top layer of the substrate). In this study, the analyte is first mixed with the nanoparticle suspension (liquid + liquid) then dried until solid (a glass slide can serve as a substrate base). This simple and new substrate/sample processing method allows encapsulating the sample inside the structure of the substrate, ensuring better interaction between the sample and gold nanoparticles and resulting in lower LODs. To the knowledge of the author, such an approach has not been used before. The advantages of this method are: a) concentration of the sample due to excess liquid extraction; b) background noise and fluorescence removal due to evaporation of the solvents from the substrate and the sample; c) low production costs; d) the ability to use the same nanoparticles suspension for numerous measurements without the need to prepare a "fresh" substrate every time (relevant to solid-support substrates).

In this study, the author presents experimentally obtained LODs for all 16 EPA priority PAHs, when at least half of the characteristic Raman peaks can be clearly observed without manipulating the spectrum. The SERS performance of a developed substrate (CNP-Au) is compared to a commercial 3D (Ram-SERS-Au) substrate. Both substrates are characterized by scanning electron microscopy imaging.

\section{Materials and Methods}

\subsection{Preparation of SERS Substrates}

A clustered gold nanoparticle suspension (CNP-Au) was prepared by Ocean Optics with the following procedure. Gold (III) chloride hydrate (HAuCl4) and sodium citrate tribasic dehydrate were purchased from Sigma-Aldrich. The colloidal gold nanoparticle suspension consists of 50 $\mathrm{nm}$ diameter gold nanospheres in water that exhibit a surface plasmon resonance (SPR) absorption band of $\sim 785$ $\mathrm{nm}$. Spherical gold nanoparticles were synthesized according to the method of Lee and Meisel [30]. Briefly, $0.8 \mathrm{ml}$ of $0.294 \mathrm{M} \mathrm{HAuCl} 4$ was added to $400 \mathrm{ml}$ of purified water and heated to a vigorous boil in a beaker. At this point, $120 \mathrm{mg}$ of sodium citrate in $1 \mathrm{ml}$ of water was added to the gold solution, where the solution changes from colorless to reddish-purple within minutes, indicating the formation of gold nanoparticles. After stirring and boiling for 15 minutes, the solution is removed from heat and allowed to cool overnight before further processing. The CNP-Au solution can be stored in the fridge up to 1 year (longer term was not experimentally tested). Before each measurement $50 \mu 1$ of CNP-Au solution is mixed with, 50 $\mu 1$ of the analyte on the top of a glass slide (or petri dish) and placed in the oven at $45^{\circ} \mathrm{C}$ for $1 \mathrm{~h}$ to allow for liquid evaporation and crystal formation.

Quartz fiber embedded gold nanoparticles (Ram-SERS-Au) were prepared by Ocean Optics according to a method developed by Hoppmann et al. Gold nanoparticles were concentrated 100 times, removing the supernatant and redispersing the pellet in a 5:4:1 nanoparticles:glycerol:ethanol mixture [31]. Next, a few microliters of the formulated ink was deposited on quartz paper substrates pre-cut into $8 \mathrm{~mm}$ diameter circles and allowed to dry. The substrates are made of $50 \mathrm{~nm}$ diameter gold nanospheres deposited on $8 \mathrm{~mm}$ diameter quartz paper circles.

\subsection{Chemicals and Solutions}

All PAHs were purchased from Sigma-Aldrich. Acenaphthene, acenaphthylene, anthracene, benzo[a]pyrene, dibenz[a,h]anthracene, fluoranthene, fluorene, Indeno[1,2,3-cd]pyrene, naphthalene, pyrene, phenanthrene and fluoranthene were in a powder state and dissolved in methanol (Fisher chemical, 99.9\%) in concentrations of $1000 \mathrm{ppm}$ (stock solution), $100 \mathrm{ppm}$ (diluted), $10 \mathrm{ppm}$ (diluted), $1 \mathrm{ppm}$ (diluted) and $0.1 \mathrm{ppm}$ (diluted). Stock solutions were made based on the following calculations: 


$$
\left.\mathrm{C}_{p p m}=10^{6} \cdot \mathrm{C}_{g / l} / \rho_{(\mathrm{kg} / \mathrm{m}}{ }^{3}\right)
$$

where $\mathrm{C}$ is concentration and $\rho$ is solution density.

Bnzo[ghi]perylene (in methylene chloride), benzo[k]fluoranthene (powder) and crysene (powder) were dissolved in acetone (from Fisher Chemical) in the same concentrations as above. Benz[a]anthracene $(1000 \mu \mathrm{g} / \mathrm{ml})$ and Benzo[b]fluoranthene $(200 \mu \mathrm{g} / \mathrm{ml})$ came already in methanol solution. After this preparation, all the solutions were stored in a freezer at $-20^{\circ} \mathrm{C}$.

\subsection{Instrumentation}

Microbalance scales from Mettler Toledo XP6 model were used to weigh PAHs.

All SERS measurements were recorded at room temperature using a commercially available Raman microscope, Xplora Plus by HORIBA France SAS. The laser beam was focused through a standard $\mathrm{x} 50$ objective with $1.9 \mu \mathrm{m}$ laser spot size. All Raman/SERS spectra were recorded with an excitation wavelength of $785 \mathrm{~nm}$, beam filter of $10 \%$, and $10 \mathrm{~mW}$ laser power. The laser beam exposure time was $10 \mathrm{~s}$, and accumulation of 10 . The intensity of the Raman peak at $520 \mathrm{~cm}^{-1}$ from silicon was normalized before each data acquisition. Data was analyzed using LabSpec 6.

\section{Results}

\subsection{Characterization of SERS Substrates}

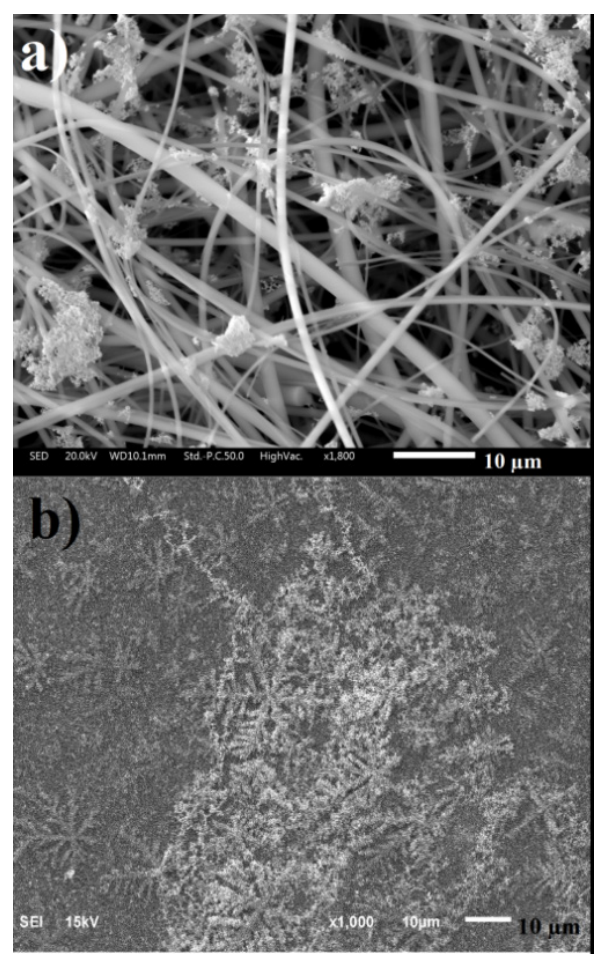

Figure 1. Scanning electron microscopy image of the surface enhanced Raman scattering substrate a) Quartz fiber embedded gold nanoparticles
(Ram-SERS-Au); b) Clustered gold nanoparticles in the solid phase (CNP-Au), prepared before each measurement by evaporating the liquid from gold nanoparticles suspension.

Scanning Electron Microscopy (SEM) images were taken to characterize the SERS surfaces. Figure 1a displays the surface morphology of the conventional solid-support RAM-SERS-Au substrate. As can be seen, gold nanoparticles are self-assembled in clusters and dispersed around quartz fibers in 3D space. Such particle distribution provides high density hot spots, thus enhancing the SERS signal. In contrast, a more efficient way to create higher density hot spot areas is to allow gold particles to self-assemble into crystals (CNP-Au substrate), which is shown in Figure 1b. All the gold particles are grouped into 3D clustered crystals, providing an enormous number of hot spots. Since the CNP-Au substrate doesn't have a uniform homogeneity, in order to achieve optimal results, the SERS measurements were performed with the laser beam focused on the crystals.

\subsection{Raman Signal Extraction}

The evaporation of the excess liquid from the sample allows strong enhancement of the Raman signal. Figure $2 \mathrm{a}$ shows Raman measurements of $1 \mathrm{ppm}$ pyrene in methanol, which is mixed with CNP-Au substrate (50:50) in a liquid form. The signal from pyrene is too weak to be detected. After the drying process, all major pyrene peaks can be clearly observed with practically no background noise (figure 2b). Four Raman peaks from pyrene at $406 \mathrm{~cm}^{-1}$ (C-C-C bending), $589 \mathrm{~cm}^{-1}$ (C-C-C bending), $1235 \mathrm{~cm}^{-1}$ (C-C stretching) and $1401 \mathrm{~cm}^{-1}$ (C-C stretching) closely correspond with that of solid pyrene (see table 1), while the $1609 \mathrm{~cm}^{-1}$ peak has been strongly shifted compared to the $1625 \mathrm{~cm}^{-1}$ of a solid pyrene. Such shifts can occur during photochemical reactions upon irradiation by the laser, when the pyrene is absorbed onto gold colloidal nanoparticles [24].

\subsection{Limits of Detection}

Since the focus of this work is to demonstrate a suitability of the suspension method for potential non-targeted analysis, it is essential to observe at least half of the characteristic Raman peaks in the sample. An example of determining LOD for one PAH, fluoranthene, at six different concentrations in methanol using conventional Ram-SERS-Au substrates and CNP-Au substrate (prepared by suspension method) is shown in figure 3. As we can observe, LOD of fluoranthene for CNP-Au (figure 3a) is an order of magnitude lower (0.1 $\mathrm{ppm}$ ) than for Ram-SERS-Au (1 ppm, figure 3b). Note, that "blank" spectra of the CNP-Au substrate (figure 3a, "0 ppm") does not produce any additional signals compared to the "blank" measurement of Ram-SERS-Au (figure 3b, "0 ppm"). This is due to the evaporation of the solvents and other chemicals during the substrate drying process. 

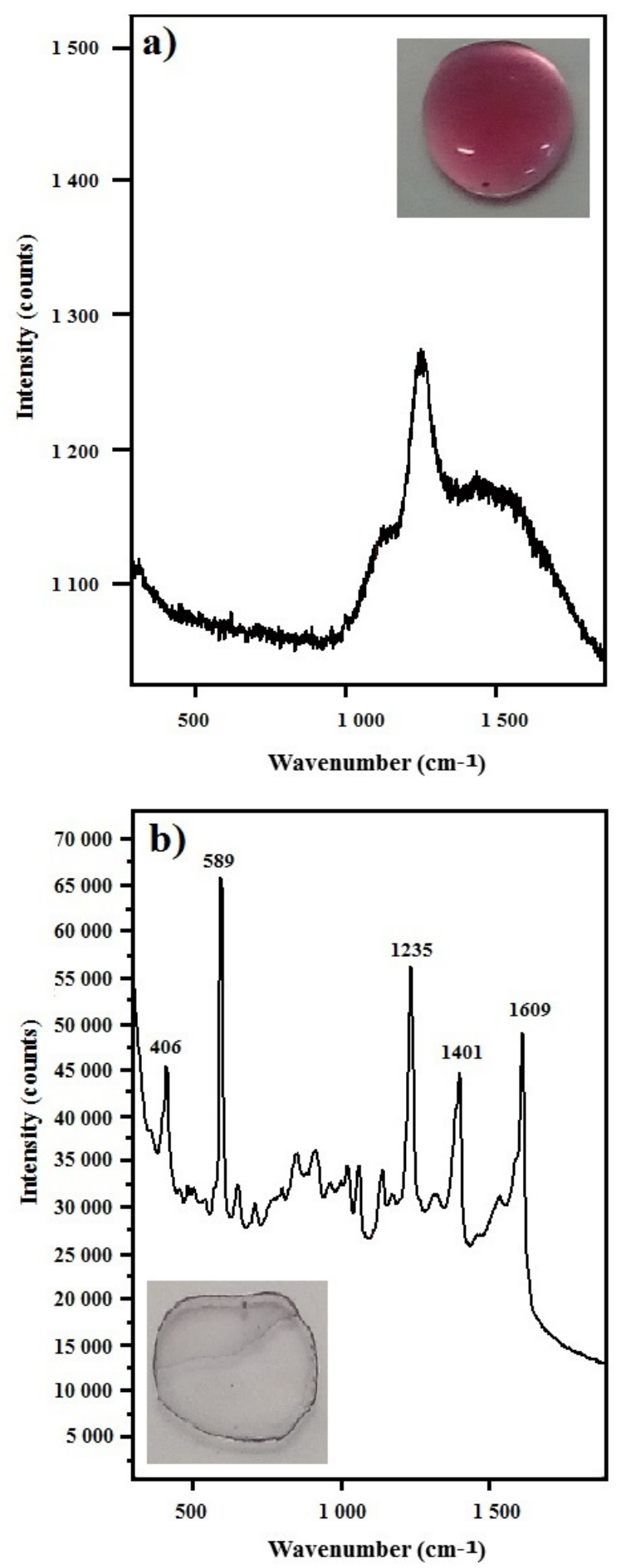

Figure 2. Spectrum of $1 \mathrm{ppm}$ pyrene in $\mathrm{MeOH}$ on CNP-Au substrate in a) liquid state, b) dried form. $\Lambda=785 \mathrm{~nm}$, laser power $10 \mathrm{~mW}$, integration time $10 \mathrm{~s}$
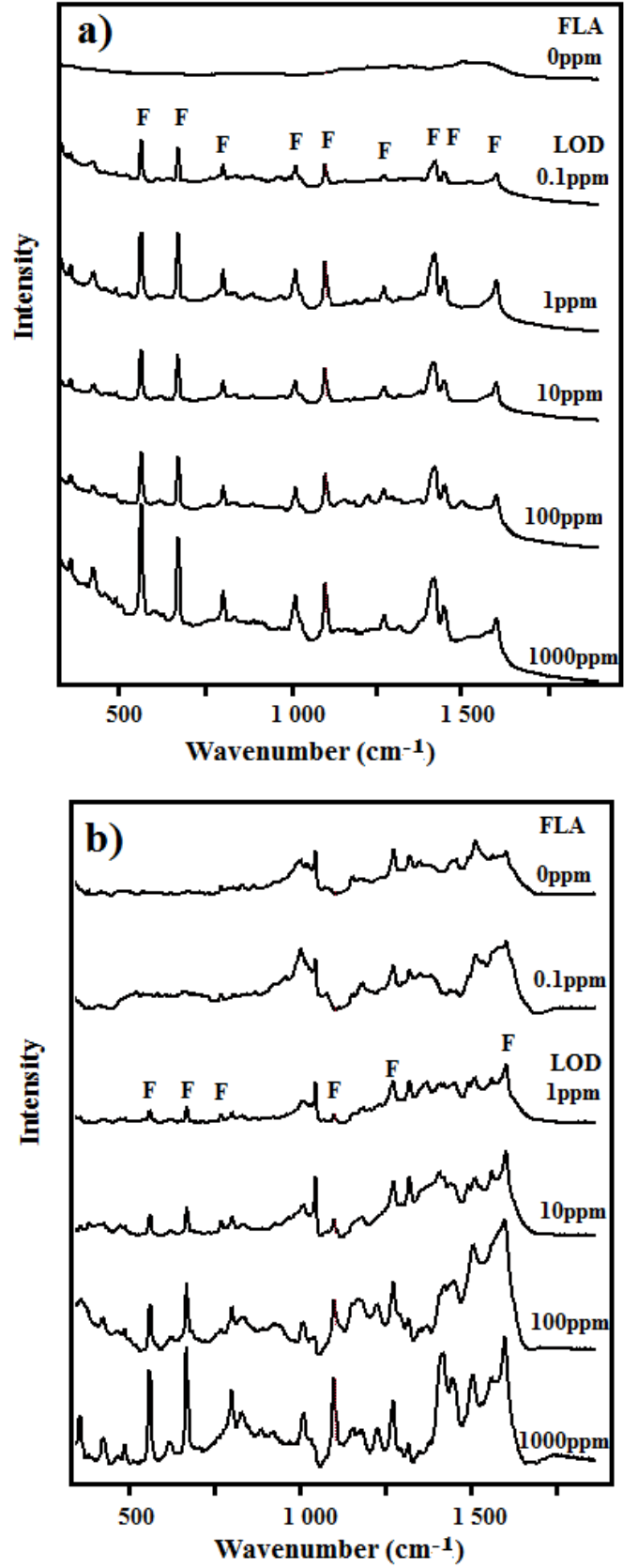

Figure 3. Spectra of fluoranthene in methanol in different concentrations between 0 ppm-1000 ppm on: a) CNP-Au (five different substrates); b) Ram-SERS-Au (same substrate). $\Lambda=785 \mathrm{~nm}$, laser power $10 \mathrm{~mW}$, integration time $10 \mathrm{~s}$. Background signal removing programs were not applied during preparation of this figure

Experimentally obtained LODs for all 16 EPA priority PAHs on conventional solid-support RAM-SERS-Au substrate and developed CNP-Au aggregation method as well as conventional Raman signals (from former studies [4]) are presented in the table below. Comparing experimental performance of the two substrates, we can see that overall LODs for CNP-Au (ranges between 0,001 ppm and $10 \mathrm{ppm}$ ) are three orders of magnitude lower than for RAM-SERS-Au (between $1 \mathrm{ppm}$ and $1000 \mathrm{ppm}$ ). In two cases RAM-SERS-Au is not sensitive for a selected chemical (acenapthene and anthracene), while CNP-Au is 
sensitive to all tested analytes. Only benzo[ghi]perylene and crysene have the same performance for both substrates ( 1 and $10 \mathrm{ppm}$, respectively), while in all other cases CNP-Au produces lower LODs. The best performance of the CNP-Au substrate compared to the Ram-SERS-Au is when measuring acenapthylene, where the sensitivity difference is four orders of magnitude. It should be noted, that SERS/Raman band shift of the same analyte on the different substrates (as well as comparing to conventional Raman) is expected, because of a chemisorption mechanism over physisorption. During chemisorption, the molecular structure of an adsorbate is modified due to the overlap of electron wave functions between analyte and metal colloids [32], resulting in slight Raman band shifts. Physisorpt molecules produce the same spectrum as free molecules except for their difference in bandwidth [9].

Table 1. Raman and SERS measurements of 16 EPA priority PAHs. Strong signals are in bold

\begin{tabular}{|c|c|c|c|c|c|}
\hline РАH & $\begin{array}{l}\text { No substrate, Raman } \\
\text { shift, } \mathbf{~ c m}^{-1}\end{array}$ & $\begin{array}{l}\text { RAM-SERS-AU, } \\
\text { Raman shift, } \mathbf{c m}^{-1}\end{array}$ & $\begin{array}{l}\text { RAM- } \\
\text { SERS-AU } \\
\text { LOD, ppm }\end{array}$ & $\begin{array}{l}\text { CNP-Au, Raman } \\
\text { shift, } \text { cm }^{-1}\end{array}$ & $\begin{array}{l}\text { CNP-Au LOD, } \\
\text { ppm }\end{array}$ \\
\hline acenaphthene & Not measured & --------------- & Not sensitive & \begin{tabular}{|llll}
600 & $\mathbf{6 4 2}$ & 961 & 984 \\
$\mathbf{1 0 1 7}$ & $\mathbf{1 2 1 4}$ & $\mathbf{1 5 8 5}$ & \\
\end{tabular} & 0,1 \\
\hline acenaphthylene & Not measured & $\begin{array}{lllll}547 & 656 & 675 & 800 & 889 \\
959 & \mathbf{9 9 9} & 1027 & 1051 \\
\mathbf{1 3 4 5} & \mathbf{1 4 2 4} & & & \end{array}$ & 1000 & $\begin{array}{lccr}\mathbf{5 4 5} & 654 & \mathbf{7 9 5} & \mathbf{9 9 9} \\
1022 & \mathbf{1 0 5 1} & \mathbf{1 3 2 5} \\
\mathbf{1 4 1 4} & & \end{array}$ & 0,1 \\
\hline anthracene & $\begin{array}{lllll}754 & 1008 & 1163 & 1186 \\
1259 & \mathbf{1 4 0 2} & 1479 & 1555 \\
\end{array}$ & -------------- & Not sensitive & $\begin{array}{l}754100611611254 \\
\mathbf{1 3 9 4} 1548\end{array}$ & 0,1 \\
\hline $\begin{array}{l}\text { benz[a] } \\
\text { anthracene }\end{array}$ & 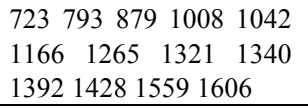 & $\begin{array}{llll}\mathbf{3 5 6} & \mathbf{7 1 8} & 787 & 1027 \\
1256 & 1337 & 1385 & 1421 \\
1549 & & & \\
\end{array}$ & 10 & 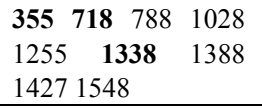 & 1 \\
\hline benzo[a]pyrene & $\begin{array}{llll}1196 & 1212 & \mathbf{1 2 3 4} & 1343 \\
\mathbf{1 3 8 4} & 1581 & 1628 & \end{array}$ & 52760912341378 & 100 & $\begin{array}{lrrr}451 & 525 & 560 & \mathbf{6 0 8} \\
632 & 753 & 84 & 901 \\
1015 & \mathbf{1 2 3 4} & 1344 \\
\mathbf{1 3 7 9} & 1569 & 1607 \\
\end{array}$ & 0,1 \\
\hline $\begin{array}{l}\text { benzo[b] } \\
\text { fluoranthene }\end{array}$ & Not measured & 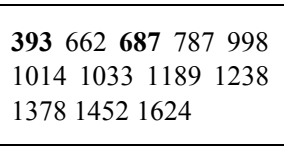 & 10 & $\begin{array}{lrrr}\mathbf{3 9 4} & 415 & 589 & 675 \\
\mathbf{6 8 4} & 783 & 993 & 1128 \\
1188 & 1339 & \mathbf{1 3 7 6} \\
1453 & 1585 & 1616 \\
\end{array}$ & 1 \\
\hline $\begin{array}{l}\text { benzo[ghi] } \\
\text { perylene }\end{array}$ & $\begin{array}{lllrl}454 & 482 & 543 & 988 & 1083 \\
1153 & 1222 & 1259 & \mathbf{1 3 0 4} \\
\mathbf{1 3 8 9} & \mathbf{1 6 0 6} & & & \\
\end{array}$ & $\begin{array}{l}3894564835411076 \\
1254130413871594\end{array}$ & 1 & $\begin{array}{lllr}385 & 413 & 453 & 479 \\
\mathbf{5 3 9} & 613 & 1074 & \mathbf{1 2 5 3} \\
\mathbf{1 3 0 2} & \mathbf{1 3 8 5} & \mathbf{1 5 9 0} \\
\end{array}$ & 1 \\
\hline $\begin{array}{l}\text { benzo[k] } \\
\text { fluoranthene }\end{array}$ & $\begin{array}{lllll}450 & 493 & 554 & 667 & 761 \\
803 & 920 & 1025 & 1093 & 1272 \\
1357 & \mathbf{1 4 4 7} & 1498 & \mathbf{1 6 0 9}\end{array}$ & $\begin{array}{lllll}452 & \mathbf{5 5 0} & 667 & 756 & 798 \\
915 & 1023 & 1087 & 1354 \\
1437 & & & \end{array}$ & 10 & $\begin{array}{rcrr}450 & \mathbf{5 5 0} & 667 & 760 \\
798 & 914 & 1019 & 1085 \\
1271 & 1360 & \mathbf{1 4 3 6} \\
1590 & & & \end{array}$ & 1 \\
\hline chrysene & $\begin{array}{lllll}598 & 677 & 772 & 1019 & \mathbf{1 3 8 3} \\
1431 & 1571 & & & \\
\end{array}$ & $\begin{array}{llll}567 & 677 & 764 & \mathbf{1 3 7 5} \\
1423 & & & \\
\end{array}$ & 1 & $\begin{array}{llll}564 & 675 & 767 & 1011 \\
\mathbf{1 3 7 5} & 1424 & 1566\end{array}$ & 0,001 \\
\hline $\begin{array}{l}\text { dibenz[ah] } \\
\text { anthracene }\end{array}$ & Not measured & $\begin{array}{llllr}426 & 494 & 545 & 618 & \mathbf{7 1 7} \\
748 & 1031 & 1215 & 1295 \\
\mathbf{1 3 4 3} & 1432 & & \\
\end{array}$ & 100 & $\begin{array}{llllr}328 & 43 & 496 & 546 & 617 \\
750 & 716 & 1030 & 1213 \\
194 & 1341 & 1427 & \\
\end{array}$ & 1 \\
\hline fluoranthene & $\begin{array}{llllll}486 & 564 & 674 & 80 & 827 \\
1022 & \mathbf{1 1 0 3} & 1136 & 1272 \\
1370 & \mathbf{1 4 2 1} & 1457 & 1609 \\
\end{array}$ & \begin{tabular}{llll|}
$\mathbf{5 6 0}$ & $\mathbf{6 6 4}$ & 795 & $\mathbf{1 0 4 1}$ \\
1095 & 1268 & 1313 & 1364 \\
1443 & 1504 & 1596 & \\
\end{tabular} & 1 & $\begin{array}{lccr}416 & \mathbf{5 6 0} & \mathbf{6 6 9} & 799 \\
1010 & \mathbf{1 0 9 7} & 1268 \\
1417 & 1449 & 1601 & \\
\end{array}$ & 0,1 \\
\hline fluorene & $\begin{array}{lllll}418 & 744 & 844 & 1022 & 1149 \\
1236 & 1291 & 1340 & 1392 \\
1473 & 1609 & & \\
\end{array}$ & $\begin{array}{llll}603 & 822 & 1000 & 1145 \\
1341 & 1550 & & \end{array}$ & 10 & $\begin{array}{|lccc|}\mathbf{7 3 8} & 783 & 862 & \mathbf{1 0 1 9} \\
1151 & \mathbf{1 2 1 9} & \mathbf{1 2 9 3} \\
1348 & 1477 & \mathbf{1 5 3 0} & \\
\end{array}$ & 10 \\
\hline $\begin{array}{l}\text { indeno[ }[1,2,3-\mathrm{cd}] \\
\text { pyrene }\end{array}$ & Not measured & $\begin{array}{l}408513 \quad 581 \quad 628 \quad 678 \\
998 \mathbf{1 2 3 7} 14181607\end{array}$ & 100 & $\begin{array}{lccr}408 & 472 & 511 & 582 \\
625 & 674 & 997 & 1185 \\
1205 & \mathbf{1 2 3 7} & \mathbf{1 4 1 6} \\
1605 & 1637 & \\
\end{array}$ & 1 \\
\hline naphthalene & $\begin{array}{l}515 \mathbf{7 6 5} 102213851460 \\
1571\end{array}$ & $\begin{array}{llll}501 & 683 & 1146 & \mathbf{1 2 6 8} \\
1381 & 1341 & 1442 & 1498\end{array}$ & 1 & $\begin{array}{llllr}391 & 503 & 565 & 646 \\
\mathbf{8 5 3} & \mathbf{8 9 8} & \mathbf{9 9 8} & 1238 \\
1317 & \mathbf{1 3 8 3} & 1495 & \\
\end{array}$ & 0,1 \\
\hline phenantrene & $\begin{array}{l}\mathbf{7 1 2} 834103911691245 \\
\mathbf{1 3 5 0} 1437\end{array}$ & $\begin{array}{llll}543 & \mathbf{7 0 6} & 827 & 1030 \\
1198 & 1268 & \mathbf{1 3 5 1} & 1428 \\
1600 & & & \\
\end{array}$ & 100 & $\begin{array}{lllr}404 & 543 & \mathbf{7 0 6} & 827 \\
1030 & 1200 & 1240 \\
1350 & 1430 & 1600\end{array}$ & 0,1 \\
\hline pyrene & $\begin{array}{l}40659610661143 \mathbf{1 2 4 2} \\
\mathbf{1 4 0 5} 15931625\end{array}$ & 40358812351617 & 1 & $\begin{array}{llll}406 & \mathbf{5 8 9} & 642 & 1016 \\
1056 & 1137 & \mathbf{1 2 3 5} \\
1401 & 1609 & \\
\end{array}$ & 0,1 \\
\hline
\end{tabular}



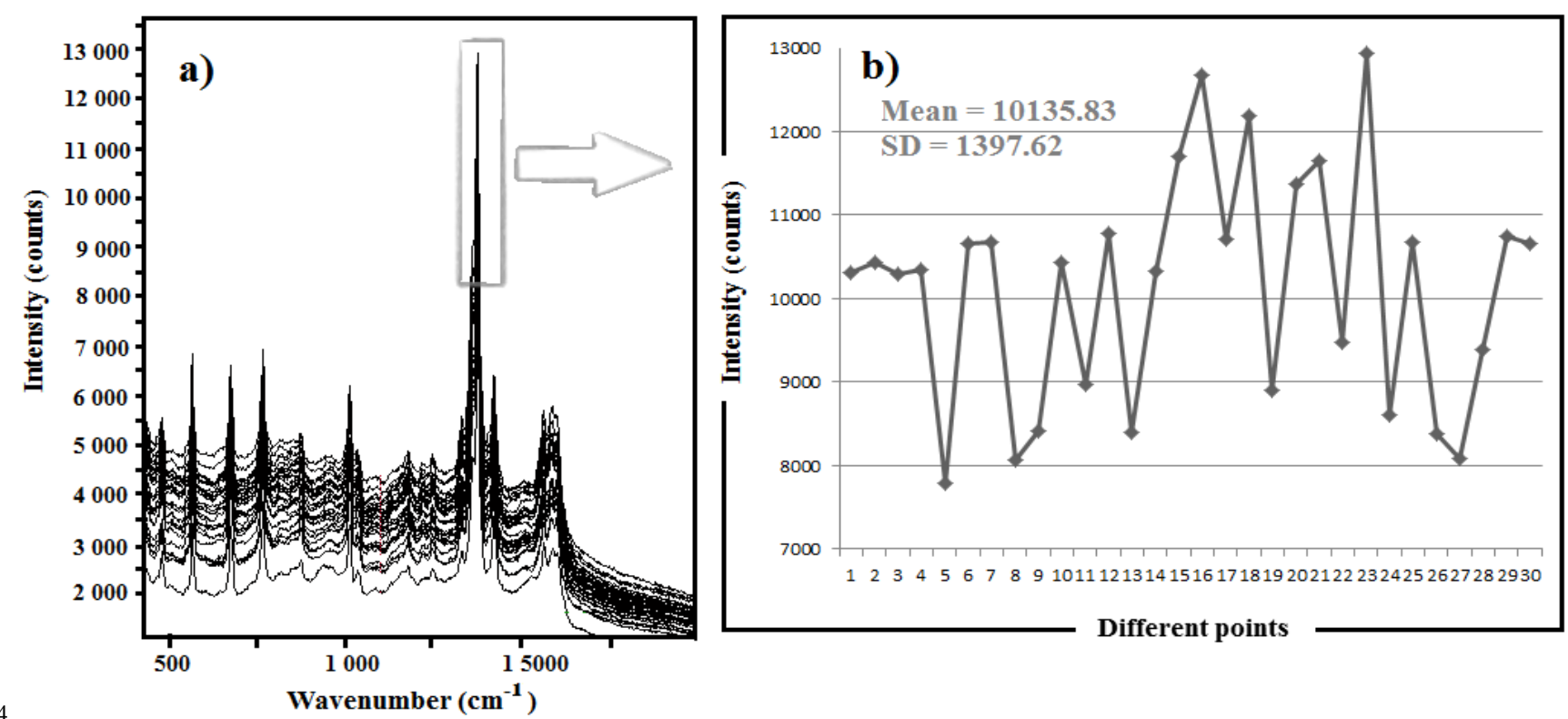

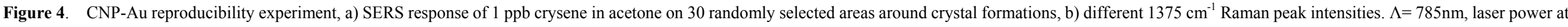
the sample $10 \mathrm{~mW}, 10 \mathrm{~s}$ integration time 


\subsection{Reproducibility}

For the purpose of determining reproducibility of SERS signal of the $1 \mathrm{ppb}$ crysene on CNP-Au substrate, it was measured 30 times in different spots of the different substrates within the area of crystal formations (figure 4a). The intensities of the Raman peaks at $1375 \mathrm{~cm}^{-1}$ are plotted in figure $4 \mathrm{~b}$, with the mean value of 10135.83 counts and standard deviation 1397.62 counts (13.78\%), which falls within the acceptable value of $20 \%$ [33].

\section{Summary and Discussion}

This study reports the application of gold nanoparticle suspensions, developed by a new method in comparison to conventional solid-support SERS substrates and is confirmed experimentally with the measurement of EPA's 16 priority PAHs. The novel method proposes prior mixing of the analyte with the substrate in a liquid form, followed by drying before each SERS measurement. This approach deceases experimental analyte detection limits up to three orders of magnitude compared to that from solid-support substrate (down to $1 \mathrm{ppb}$ ), as well as complete elimination of the background signal from the substrate. Reproducibility of the measurements is high (STD is below 14\%). Apart from the advantages already listed, the application of this method is remarkably cost efficient due to the small amount of substrate needed for each measurement. This method does not replace previous studies, but offers a complimentary technique that can be used to attain a Raman signal in untargeted samples.

\section{Limitations}

The application of the described method has certain limitations. Due to a nonhomogeneous surface morphology, dried nanoparticles suspensions are not quantitative. Also it cannot be used for thermally unstable samples and in-situ applications may be limited.

\section{Acknowledgements}

The author gratefully acknowledges Prof. Chelsea Rochman from University of Toronto for professional support and allowing to use the laboratory and the Raman instrument; Prof. David Sinton (also from University of Toronto) for providing chemicals and consumables for the current study; Anne-Marie Dowgiallo from Ocean Optics for preparation of RAM-SERS-Au and CNP-Au substrates; as well as Jelena Grbic and James Chalmers for editing support.

\section{REFERENCES}

[1] H. I. Abdel-Shafy and M. S. M. Mansour, "A review on polycyclic aromatic hydrocarbons: Source, environmental impact, effect on human health and remediation," Egyptian Journal of Petroleum. 2016.

[2] J. R. Ferraro, K. Nakamoto, and C. W. Brown, Introductory Raman Spectroscopy. 2003.

[3] S. Lucht, T. Murphy, H. Schmidt, and H.-D. Kronfeldt, "Optimized recipe for sol-gel-based SERS substrates," $J$. Raman Spectrosc., vol. 31, no. 11, pp. 1017-1022, Nov. 2000.

[4] A. Kolomijeca, "An autonomous sea going Raman/SERS instrument for in situ detection of chemicals in sea water (Book, 2013) [WorldCat.org]," Technical University Berlin, 2013.

[5] S.-Y. Ding, E.-M. You, Z.-Q. Tian, and M. Moskovits, "Electromagnetic theories of surface-enhanced Raman spectroscopy," Chem. Soc. Rev., vol. 46, no. 13, pp. 4042 4076, Jul. 2017.

[6] A. Shiohara, Y. Wang, and L. M. Liz-Marzán, "Recent approaches toward creation of hot spots for SERS detection," J. Photochem. Photobiol. C Photochem. Rev., vol. 21, pp. 2-25, Dec. 2014

[7] P. Mosier-Boss, Mosier-Boss, and P. A., "Review of SERS Substrates for Chemical Sensing," Nanomaterials, vol. 7, no. 6 , p. 142, Jun. 2017.

[8] F. Hu et al., "Smart Liquid SERS Substrates based on $\mathrm{Fe} 3 \mathrm{O} 4 / \mathrm{Au}$ Nanoparticles with Reversibly Tunable Enhancement Factor for Practical Quantitative Detection," Sci. Rep., vol. 4, no. 1, p. 7204, May 2015.

[9] Y.-H. Kwon, K. Sowoidnich, H. Schmidt, and H.-D. Kronfeldt, "Application of calixarene to high active surface-enhanced Raman scattering (SERS) substrates suitable for in situ detection of polycyclic aromatic hydrocarbons (PAHs) in seawater," J. Raman Spectrosc., vol. 43, no. 8, pp. 1003-1009, Aug. 2012.

[10] A. Kolomijeca, Y.-H. Kwon, K. Sowoidnich, R. D. Prien, D. E. Schulz-Bull, and H.-D. Kronfeldt, "High sensitive Raman sensor for continuous in-situ detection of PAHs," in Proceedings of the International Offshore and Polar Engineering Conference, 2011.

[11] C. L. H. and and R. P. Van Duyne*, "Nanosphere Lithography: A Versatile Nanofabrication Tool for Studies of Size-Dependent Nanoparticle Optics,” 2001.

[12] G. A. Baker and D. S. Moore, "Progress in plasmonic engineering of surface-enhanced Raman-scattering substrates toward ultra-trace analysis," Anal. Bioanal. Chem., vol. 382, no. 8, pp. 1751-1770, Aug. 2005.

[13] R. Ossig, A. Kolomijeca, Y.-H. Kwon, F. Hubenthal, and H.-D. Kronfeldt, "SERS signal response and SERS/SERDS spectra of fluoranthene in water on naturally grown $\mathrm{Ag}$ nanoparticle ensembles," J. Raman Spectrosc., vol. 44, no. $5,2013$.

[14] L. Wu et al., "Highly sensitive, reproducible and uniform SERS substrates with a high density of three-dimensionally distributed hotspots: gyroid-structured Au periodic metallic materials," NPG Asia Mater., vol. 10, no. 1, p. e462, Jan. 2018. 
[15] A. Kolomijeca, H. D. Kronfeldt, and Y. H. Kwon, "A portable Surface Enhanced Raman Spectroscopy (SERS) Sensor System applied for seawater and sediment investigations on an Arctic sea-trial," Int. J. Offshore Polar Eng., 2013.

[16] Y. H. Kwon, A. Kolomijeca, H. D. Kronfeldt, R. Ossig, and F. Hubenthal, "Naturally grown Ag nanoparticle SERS substrate as chemical sensor in fresh water applying $488 \mathrm{~nm}$ microsystem laser diode," Int. J. Offshore Polar Eng., 2013.

[17] O. Péron, E. Rinnert, M. Lehaitre, P. Crassous, and C. Compère, "Detection of polycyclic aromatic hydrocarbon (PAH) compounds in artificial sea-water using surface-enhanced Raman scattering (SERS)," Talanta, vol. 79, no. 2, pp. 199-204, Jul. 2009.

[18] O. Péron, E. Rinnert, T. Toury, M. Lamy de la Chapelle, and C. Compère, "Quantitative SERS sensors for environmental analysis of naphthalene," Analyst, vol. 136, no. 5, pp. 1018-1022, Feb. 2011.

[19] H. Schmidt, N. Bich Ha, J. Pfannkuche, H. Amann, H.-D. Kronfeldt, and G. Kowalewska, "Detection of PAHs in seawater using surface-enhanced Raman scattering (SERS)," Mar. Pollut. Bull., vol. 49, no. 3, pp. 229-234, Aug. 2004.

[20] L. G. Olson, R. H. Uibel, and J. M. Harris, "C18-Modified Metal-Colloid Substrates for Surface-Enhanced Raman Detection of Trace-Level Polycyclic Aromatic Hydrocarbons in Aqueous Solution," Appl. Spectrosc., vol. 58, no. 12, pp. 1394-1400, Dec. 2004.

[21] L. Bao, P. Sheng, J. Li, S. Wu, Q. Cai, and S. Yao, "Surface enhanced Raman spectroscopic detection of polycyclic aromatic hydrocarbons (PAHs) using a gold nanoparticles-modified alginate gel network," Analyst, vol. 137, no. 17, p. 4010, Jul. 2012.

[22] X. Jiang, Y. Lai, M. Yang, H. Yang, W. Jiang, and J. Zhan, "Silver nanoparticle aggregates on copper foil for reliable quantitative SERS analysis of polycyclic aromatic hydrocarbons with a portable Raman spectrometer," Analyst, vol. 137, no. 17, p. 3995, Jul. 2012.

[23] Y. Xie et al., "Selective SERS detection of each polycyclic aromatic hydrocarbon (PAH) in a mixture of five kinds of PAHs," J. Raman Spectrosc., vol. 42, no. 5, pp. 945-950, May 2011.

[24] X. Shi, S. Liu, X. Han, J. Ma, Y. Jiang, and G. Yu, "High-Sensitivity Surface-Enhanced Raman Scattering (SERS) Substrate Based on a Gold Colloid Solution with a $\mathrm{pH}$ Change for Detection of Trace-Level Polycyclic Aromatic Hydrocarbons in Aqueous Solution," Appl. Spectrosc., vol. 69, no. 5, pp. 574-579, May 2015.

[25] X. Shi, J. Ma, R. Zheng, C. Wang, and H.-D. Kronfeldt, "An improved self-assembly gold colloid film as surface-enhanced Raman substrate for detection of trace-level polycyclic aromatic hydrocarbons in aqueous solution," J. Raman Spectrosc., vol. 43, no. 10, pp. 1354 1359, Oct. 2012.

[26] H. Wang, J. Zhao, A. M. D. Lee, H. Lui, and H. Zeng, "Improving skin Raman spectral quality by fluorescence photobleaching," Photodiagnosis Photodyn. Ther., vol. 9, no. 4, pp. 299-302, Dec. 2012.

[27] K. Otto, C. P. Hubbard, W. H. Weber, and G. W. Graham,
"Raman spectroscopy of palladium oxide on $\gamma$-alumina applicable to automotive catalysts: Nondestructive, quantitative analysis; oxidation kinetics; fluorescence quenching," Appl. Catal. B Environ., vol. 1, no. 4, pp. $317-$ 327, Dec. 1992.

[28] S. Yang et al., "Laser Wavelength Dependence of Background Fluorescence in Raman Spectroscopic Analysis of Synovial Fluid from Symptomatic Joints.," $J$. Raman Spectrosc., vol. 44, no. 8, pp. 1089-1095, Aug. 2013.

[29] J. Register, M. Maiwald, A. Fales, P. Strobbia, B. Sumpf, and T. Vo-Dinh, "Shifted-excitation Raman difference spectroscopy for the detection of SERS-encoded gold nanostar probes," J. Raman Spectrosc., vol. 49, no. 12, pp. 1961-1967, Dec. 2018.

[30] P. C. Lee and D. Meisel, "Adsorption and surface-enhanced Raman of dyes on silver and gold sols," J. Phys. Chem., vol. 86, no. 17, pp. 3391-3395, Aug. 1982.

[31] E. P. Hoppmann, W. W. Yu, and I. M. White, "Highly sensitive and flexible inkjet printed SERS sensors on paper," Methods, vol. 63, no. 3, pp. 219-224, Oct. 2013.

[32] A. Campion and P. Kambhampati, "Surface-enhanced Raman scattering," Chem. Soc. Rev., vol. 27, no. 4, p. 241, Jan. 1998

[33] U. S. Dinish, F. C. Yaw, A. Agarwal, and M. Olivo, "Development of highly reproducible nanogap SERS substrates: Comparative performance analysis and its application for glucose sensing," Biosens. Bioelectron., vol. 26, no. 5, pp. 1987-1992, Jan. 2011. 